\title{
ELT-1, a GATA-like transcription factor, is required for epidermal cell fates in Caenorhabditis elegans embryos
}

\author{
Barbara D. Page, ${ }^{1,2}$ Wei Zhang, ${ }^{1,2}$ Keith Steward, ${ }^{3}$ Tom Blumenthal, ${ }^{3,4}$ and James R. Priess ${ }^{1,2,5}$ \\ ${ }^{1}$ Fred Hutchinson Cancer Research Center (FHCRC) and ${ }^{2}$ Howard Hughes Medical Institute, Seattle, Washington 98109 \\ USA; ${ }^{3}$ Department of Biology, University of Indiana, Bloomington, Indiana 47405 USA
}

\begin{abstract}
Epidermal cells are generated during Caenorhabditis elegans embryogenesis by several distinct lineage patterns. These patterns are controlled by maternal genes that determine the identities of early embryonic blastomeres. We show that the embryonically expressed gene elt-1, which was shown previously to encode a GATA-like transcription factor, is required for the production of epidermal cells by each of these lineages. Depending on their lineage history, cells that become epidermal in wild-type embryos become either neurons or muscle cells in elt-1 mutant embryos. The ELT-1 protein is expressed in epidermal cells and in their precursors. We propose that elt-1 functions at an early step in the specification of epidermal cell fates.
\end{abstract}

[Key Words: C. elegans; hypodermal cells; cell fate specification; lineage patterns; GATA transcription factor] Received April 9, 1997; revised version accepted May 15, 1997.

Recent studies have identified some of the maternally expressed genes that pattern the early Caenorhabditis elegans embryo (for review, see Kemphues and Strome 1997; Schnabel and Priess 1997). However, there is very little molecular information about the embryonically expressed genes that presumably are regulated by these maternal genes and that lead to tissue-specific differentiation. In the present study, we provide evidence that the embryonically expressed gene elt-1 functions in the specification of a major tissue in C. elegans, the epidermis.

The epidermis consists of a simple layer of cells, one cell thick. The apical surface of each cell is encircled by an adherens junction, so that adjacent cells are linked together; the basal surface overlies a basement membrane. Historically, nematode epidermal cells have been called hypodermal cells because an extracellular cuticle covers the apical surface of these cells. In C. elegans, hypodermal cells arise from several different branches of the embryonic lineage. Both blastomeres, $\mathrm{AB}$ and $\mathrm{P}_{1}$, of the two-cell-stage embryo produce several hypodermal cells. The lineage patterns by which $\mathrm{AB}$ and $\mathrm{P}_{1}$ produce hypodermal cells, however, are very different. Whereas most of the nonhypodermal descendants of $A B$ become neurons or pharyngeal cells, most of the nonhypodermal descendants of $\mathrm{P}_{1}$ become intestinal cells or body-wall muscle cells.

${ }^{4}$ Present address: Department of Biochemistry, Biophysics, and Genetics, University of Colorado School of Medicine, Denver, Colorado 80262 USA.

${ }^{5}$ Corresponding author.

E-MAIL jpriess@fred.fhcrc.org; FAX (206) 667-3650.
Genetic and experimental studies have shown that the early molecular events that lead to hypodermal specification of $P_{1}$ descendants are different from the events that specify hypodermis from $\mathrm{AB}$. The maternally expressed gene pal-1 is required for $P_{1}$ to produce hypodermal cells as well as other cell types (Hunter and Kenyon 1996). In contrast, pal-1|+| is not required for AB descendants to produce hypodermal cells. pal-1 encodes a transcription factor with sequence similarity to Drosophila CAUDAL (Waring and Kenyon 1991), and the PAL-1 protein is present in the nuclei of $P_{1}$ descendants, but not AB descendants (Hunter and Kenyon 1996).

The hypodermal fates of $A B$ descendants are determined by a combination of cell-cell interactions (Priess et al. 1987; Gendreau et al. 1994; Hutter and Schnabel 1994; Mango et al. 1994; Mello et al. 1994; Hutter and Schnabel 1995). During the two-cell stage of embryogenesis, the $\mathrm{P}_{1}$ blastomere appears to influence the development of the $\mathrm{AB}$ blastomere such that $\mathrm{AB}$ acquires the ability to produce both hypodermal and neuronal cell types (Hutter and Schnabel 1995). In the next few cell divisions, two additional cell-cell interactions determine which specific $A B$ descendants will produce predominantly neurons and which will produce predominantly hypodermal cells. These latter two interactions are both mediated by maternal expression of the $g l p-1$ gene (Priess and Thomson 1987; Hutter and Schnabel 1994; Mello et al. 1994; Moskowitz et al. 1994). The GLP-1 protein is a transmembrane receptor similar to Drosophila NOTCH and C. elegans LIN-12 (Yochem and Greenwald 1989). GLP-1 is expressed in $A B$ and its daughters, but not in $\mathrm{P}_{1}$ or in $\mathrm{P}_{1}$ daughters (Evans et al. 
1994). Thus, the production of hypodermal cells by $A B$ and $P_{1}$ is influenced initially by distinct genetic pathways involving GLP-1 and PAL-1, respectively.

Maternally expressed genes like $g l p-1$ and pal-1 define identities of early embryonic blastomeres; they initiate the complex patterns of cell division and differentiation that distinguish the blastomeres. These maternal genes must directly or indirectly activate new transcription, leading to blastomere-specific expression of the embryonic genome. In this report we show that mutations in the elt-1 gene, which was shown previously to encode a GATA-like transcription factor of unknown function (Spieth et al. 1991), is required for the embryo to produce hypodermal cells. We find that the ELT-1 protein is expressed in the early embryo in both $A B$ and $P_{1}$-derived hypodermal precursors. We show that cells that produce hypodermis in wild-type embryos produce nonhypodermal cell types in elt-1 mutant embryos, and that the specific fates of these transformed cells depend on whether they are descendants of $\mathrm{AB}$ or $\mathrm{P}_{1}$. Thus, elt-1 identifies a point of convergence between the initially distinct pathways $\mathrm{AB}$ and $\mathrm{P}_{1}$ use to produce hypodermal cells.

\section{Background}

C. elegans embryogenesis begins with a period of rapid cell proliferation. Near the end of this period, cells on the dorsal and dorsal-lateral surfaces of the embryo become visibly different from all other cells (Fig. 1 A). The dorsal cells cease division, flatten and spread, and become linked through adherens junctions (Fig. 1B). These cells differentiate as hypodermal cells. Cells on the ventral surface of the embryo undergo one to three additional divisions; almost all of these cells differentiate as neurons or neuronal support cells (Fig. 1A). The hypodermal cells spread ventrally (Fig. 1C) and anteriorly until they enclose the embryo, thus forming a continuous epithelial surface called the hypodermis.

Two basic types of hypodermal cells, major and minor, have been described (Gendreau et al. 1994). The major and minor classes were used originally to distinguish between AB-derived descendants. All $\mathrm{P}_{1}$-derived hypodermal cells, however, have characteristics of the major class, and, in this paper, are included in the major class. There are 71 major hypodermal cells; these are large cells that are born at the 9 th round of embryonic cleavage and that cover most of the body surface. There are 11 minor hypodermal cells; these are relatively small cells that are born at the 10th round of cleavage and that form specialized syncytia at the head and tail. Analysis of the $C$. elegans cell lineage has shown that major hypodermal cells originate from four blastomeres in the 12-cell-stage embryo (Fig. 1D; Sulston et al. 1983). The AB-derived descendants are ABarp, ABpla, and ABpra; the $\mathrm{P}_{1}$-derived descendant is $\mathrm{C}$. Each of these blastomeres undergoes an additional five rounds of cell division before generating major hypodermal cells.
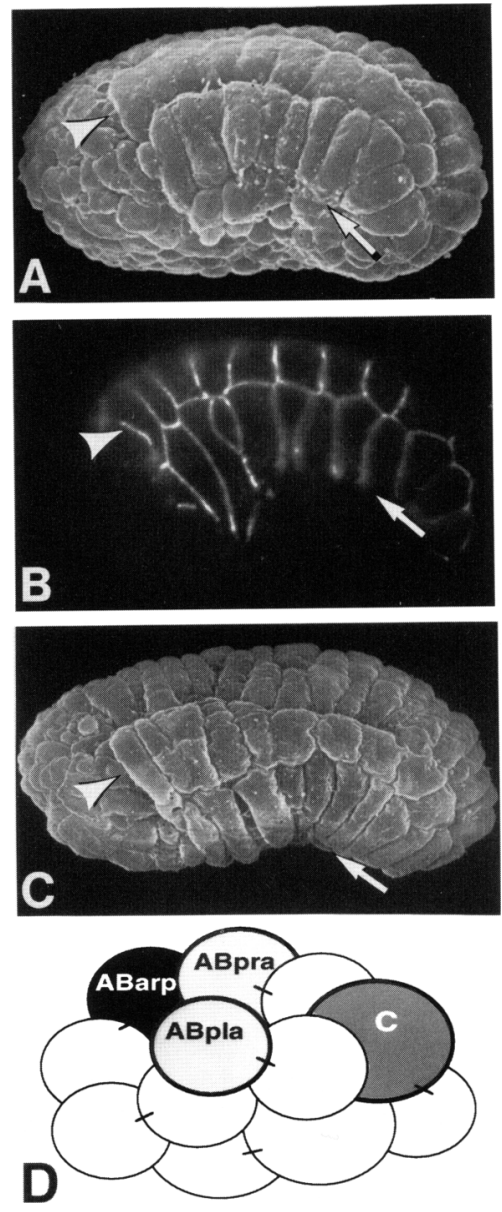

Figure 1. Positions and origins of the major hypodermal cells. (A) Scanning electron micrograph of the lateral surface of an embryo about 270 min postfertilization. The large rectangular cells on the dorsal (top) and dorsolateral surfaces are major hypodermal cells. The arrow points to the leading edge of one of several cells in the ventralmost row of hypodermal cells; these cells are in the process of spreading ventrally. The arrowhead indicates the anterior-most cell in the row of lateral, or seam, hypodermal cells. Surface cells anterior (left) and ventral (below) of the hypodermal cells are predominantly neuronal precursors that will be covered as the hypodermal cells spread across and enclose the embryo. $(B)$ Immunofluorescence micrograph of an embryo stained with mAb MH27 to visualize adherens junctions. The age, orientation, and labeling of the embryo is the same as in $A$. Note that adherens junctions are present only at points of contact between hypodermal cells. (C) Embryo at 310 min postfertilization after the hypodermal cells have enclosed the ventral, but not anterior, body surface. The labeling and orientation of the embryo are as in $A$. (D) Diagram of a 12-cell stage embryo indicating the four hypodermal precursors described in this paper; each of these blastomeres produces large numbers of hypodermal cells in addition to other cell types (Sulston et al. 1983). Embryos are $\sim 45 \mu \mathrm{m}$ in length.

\section{Results}

\section{Isolation of elt-1 mutations}

In screens for recessive, embryonic-lethal mutations affecting tissue development, we found three mutants, 
$z u 180$, zu220, and zu228, that appeared by light microscopy to have no hypodermal cells but more neurons than wild-type embryos. Genetic analysis showed that all three mutants are defective in the same gene (see Materials and Methods). The zu180 mutation was mapped to chromosome IV between fem-3 and daf-14 (Fig. 2). Cosmids covering this region were obtained from the C. elegans Genome Project and were tested for their ability to rescue the zu180 lethality (see Materials and Methods). We found that each of the overlapping cosmids W03E7, T01G2, and T12F9 has rescuing activity. Analysis of the rescuing DNA fragments showed that each contains a gene called elt-1. Because no mutations in elt-1 had been described previously, we tested whether the zu180 embryonic phenotype could be phenocopied by injection of antisense elt-1 RNA into the gonads of wild-type hermaphrodites (Guo and Kemphues 1995). The injected hermaphrodites produced inviable embryos that appeared identical to $z u 180$ mutants (see below), indicating that zu180 was likely to be a mutation in the elt-1 gene.

elt-1 was identified initially by its similarity to genes that encode GATA-like, zinc-finger transcription factors (Spieth et al. 1991). This family of transcription factors has been implicated in the specification of tissue type in vertebrates (Orkin 1992). The predicted ELT-1 protein contains two possible zinc finger domains and is $60 \%$ identical in these domains to vertebrate GATA factors, such as GATA-1, 2, and 3 from mouse. We sequenced the elt-1 gene from the zu180 strain and found that it contains a stop codon that would truncate the predicted ELT-1 protein after the first finger domain, confirming that $z u 180$ is a mutation in elt-1.

\section{elt-1 mutants lack hypodermal cells}

Homozygous elt-1 mutants do not undergo body morphogenesis (Fig. 3, cf. D with C), a process that normally requires hypodermal cell function (Sulston et al. 1983; Priess and Hirsh 1986). Major hypodermal cells can be identified on the surface of a wild-type embryo by their large, flat nuclei and prominent nucleoli (Fig. 3C,E). An elt-1 mutant contains few, if any, candidates for being hypodermal cells by these morphological criteria. Instead, the surface of an elt-1 mutant is covered with numerous cells that, by light microscopy, resemble neurons (Fig. 3D,F). Internal tissues in an elt-1 mutant, such as the pharynx and intestine, appear to contain the normal numbers and types of cells (Fig. 3, cf. B with A). Other cell types such as neurons, muscle cells, (see below) and germ cells (data not shown) also are present in an elt-1 mutant. Therefore, mutations in the elt-1 appear to cause a loss of hypodermal cells, but do not affect the ability of the embryo to produce several other tissue types.

We examined elt-1 mutant embryos with molecular markers to confirm the absence of hypodermal cells. In wild-type embryos, adherens junctions surround the apical margins of hypodermal cells, intestinal cells, and some pharyngeal cells; these adherens junctions can be visualized by staining with the antibody $\mathrm{MH} 27$ (Priess and Hirsh 1986; Waterson 1988). Wild-type and elt-1 mutant embryos appear to have identical pharyngeal and intestinal MH27 staining patterns (Fig. 3, cf. G with H). However, whereas wild-type embryos have $\sim 60$ hypodermal cells that stain with MH27 (Fig. 3I), elt-1 mutant embryos contain few or no cells with a hypodermal-like staining pattern (Fig. 3J). In elt-1 mutants, the few nonpharyngeal or nonintestinal cells that show MH27 staining are small cells of undetermined identity (see Discussion). For a second hypodermal marker, we used the lacZ fusion construct $w I s 1$ that is expressed in wild-type embryos in the lateral group of major hypodermal cells; these 20 cells are called seam cells (Gendreau et al. 1994; see Materials and Methods). We found that elt 1 mutants

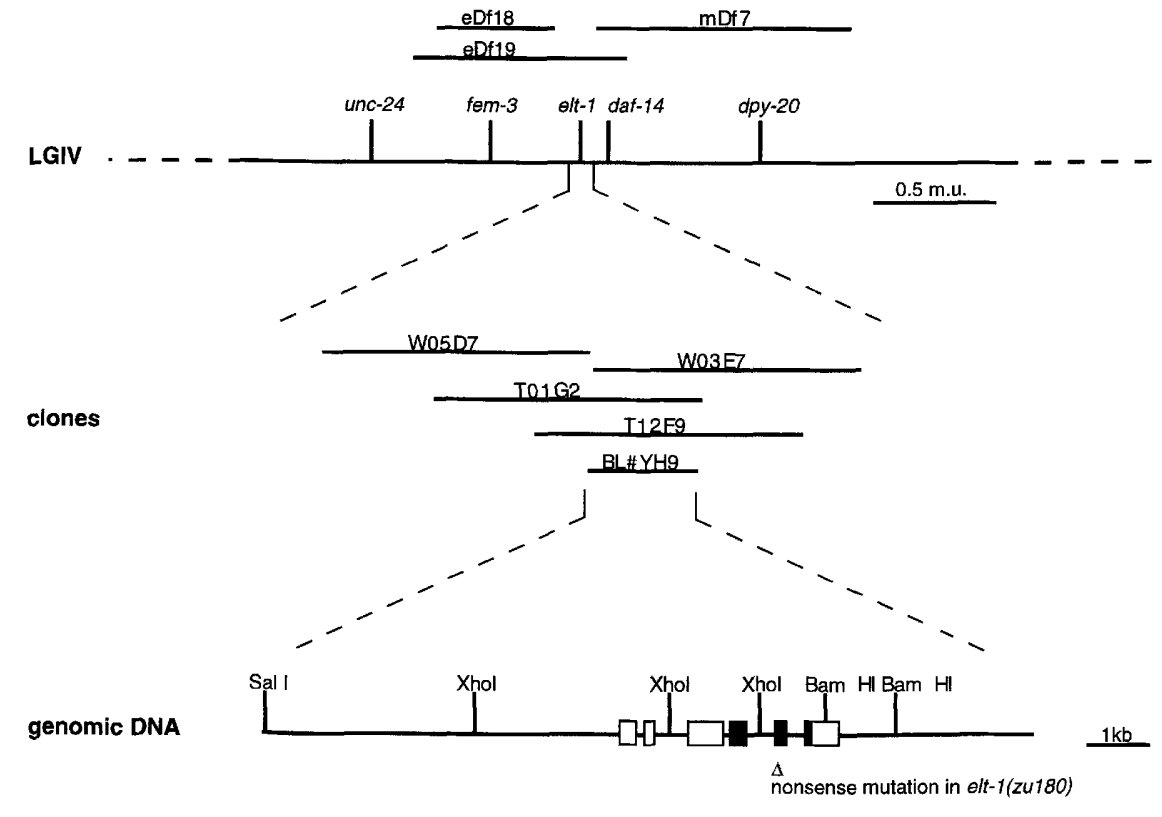

Figure 2. Molecular cloning of elt-1. The zu180 mutation maps near the elt-1 gene on chromosome IV and is complemented by the chromosomal deficiencies eDf18 and mDf7, but not by eDf19. zu180 mutants were rescued with the cosmids W03E7, T01G2, T12F9, and the phage BL\#YH9; all of which contain elt-1. Rescue was not obtained with W05D7 that does not contain the elt-1 gene. The elt-1 gene is oriented $5^{\prime}$ to $3^{\prime}$; exons are boxed; regions encoding zinc-finger domains are shaded. zu180 is a nonsense mutation in tryptophan 283. Map of elt-1 is from Spieth et al. (1991). 

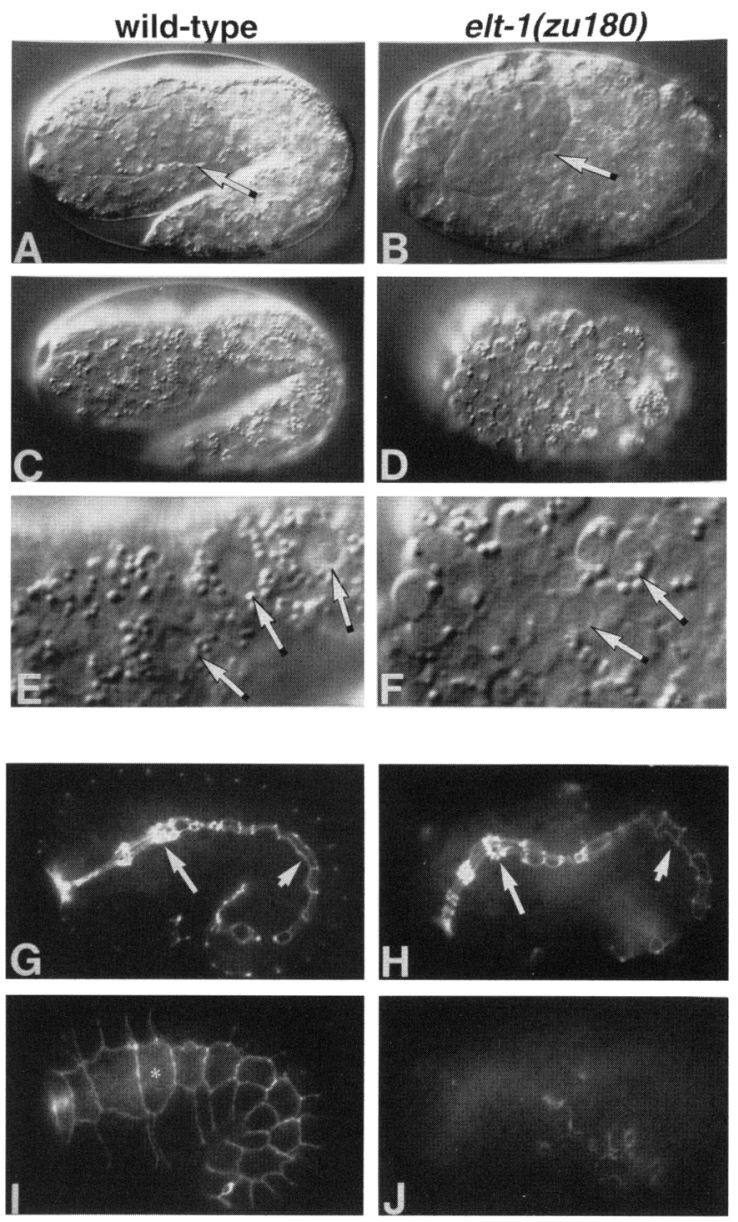

Figure 3. Comparison of wild-type embryos (left) with elt-1 mutant embryos (right). $(A-F)$ Nomarski photomicrographs taken at focal planes through the interior $(A, B)$, and at the surface $(C, D)$ of each embryo. Arrows in $A$ and $B$ indicate the basement membrane that surrounds the pharynx. $\langle E, F\rangle$ High magnification views of the surfaces of embryos shown in $C$ and $D$, respectively. Hypodermal cells have large nuclei with clear nucleoplasm and prominent nucleoli; several hypodermal nuclei are indicated in $E$ (arrows). Neurons are small cells that have processes and granular nucleoplasm; cells with neuronal morphology are indicated in $F$ (arrows). $(G-l)$ Immunofluorescence micrographs showing adherens junctions in the interiors $(G, H)$ and surfaces $(I, J)$ of fixed embryos after staining with $\mathrm{mAb}$ MH27. $(G, H)$ Long arrows point to adherens junctions in the corpus of the pharynx (Albertson and Thomson 1976); short arrows point to adherens junctions in the intestine. Cell margins of the individual major hypodermal cells are apparent at the surface of the wild-type embryo $(I)$; a hypodermal cell is indicated by the asterisk. Dots along the dorsal perimeter of the embryo in $G$ correspond to optical cross-sections through the adherens junctions of the hypodermal cells. In the elt-1 embryo $(J)$, no major hypodermal cells are visible; however, a few small cells show MH27 staining. The wild-type embryo shown in $A$ and $C$ was allowed to develop for $7 \mathrm{hr}$ at $22^{\circ} \mathrm{C}$, at which time the surface is covered by major hypodermal cells that are differentiated. The elt- 1 embryo in $B$ and $D$ developed for $12 \mathrm{hr}$ to allow the surface cells to finish division and differentiation. Embryos shown in $G-J$ were each allowed to develop for $6 \mathrm{hr}$. carrying this marker do not have detectable lac $Z$ expression (data not shown). Thus, we conclude that elt-1 mutants have a defect in either the differentiation or specification of hypodermal cells.

elt-1 mutant embryos appear by light microscopy to contain more neurons than are visible in wild-type embryos. To extend this observation, we stained elt-1 mutants with the neuron-specific antibody anti-UNC-33; this antibody stains the cytoplasm of apparently all classes of neuronal cells (J. Shaw, pers. comm.). Although we could not accurately count the numerous cells stained with anti-UNC-33 in whole-mount preparations, the elt-1 embryos (Fig. 4B) appear to have many more positively stained cells than do wild-type embryos (Fig. 4A). This result, combined with the lack of hypodermal markers in elt-1 mutants, suggests that loss of elt-1 activity may cause a transformation of some hypodermal precursors into neuronal precursors.

\section{ABa-derived hypodermal precursors become neuronal precursors in elt-1 embryos}

Twelve of the 20 seam hypodermal cells are produced by an AB descendant called ABarp (Sulston et al. 1983; Fig. 1). Because these hypodermal cells are not detectable by seam cell-specific lac $Z$ expression in elt-1 mutants, we examined the development of the ABarp blastomere by light microscopy and video recording. Results of this lineage analysis indicate that the cell division pattern of ABarp in elt-1 mutants is different from the wild-type pattern (Fig. 5A). The first difference we observe between the wild-type and elt-1 lineage patterns occurs $\sim 220 \mathrm{~min}$ postfertilization. In wild-type embryos, four ABarp descendants undergo unequal divisions in which the large
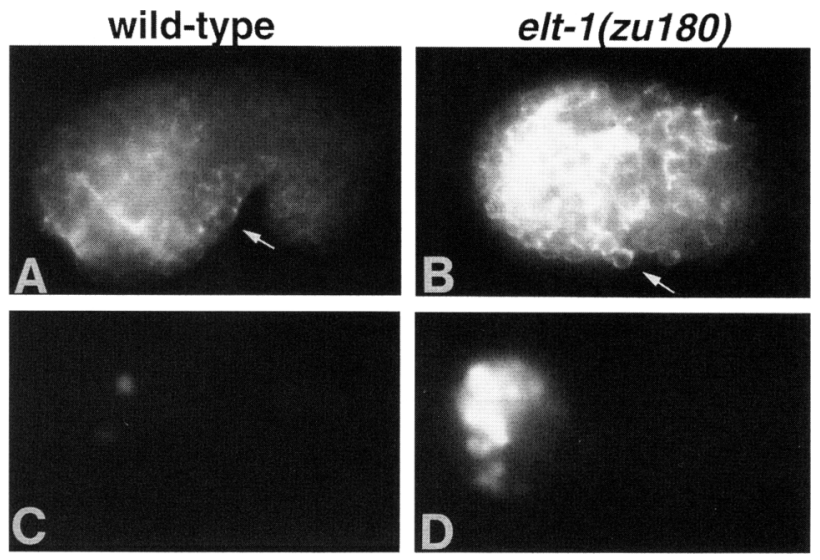

Figure 4. Neurons in wild-type (left) and elt-1 mutant embryos (right). $(A, B)$ Immunofluorescence micrographs of wild-type and elt-1 mutant embryos that developed for $6 \mathrm{hr}$ at $22^{\circ} \mathrm{C}$ before fixation and staining with the neuronal marker anti-UNC-33. $(C, D)$ Expression of the neuronal marker $\mathrm{H} 2 \mathrm{O}-\mathrm{GFP}$ in wild-type $(C)$ and elt 1 mutant $(D)$ embryos in which the only blastomeres allowed to develop were ABarp and ABalp /see Materials and Methods). 


\section{A}

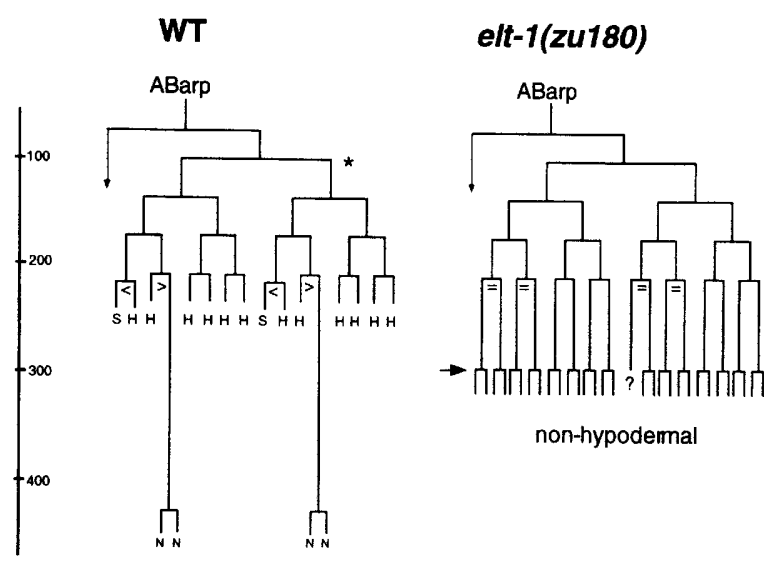

B
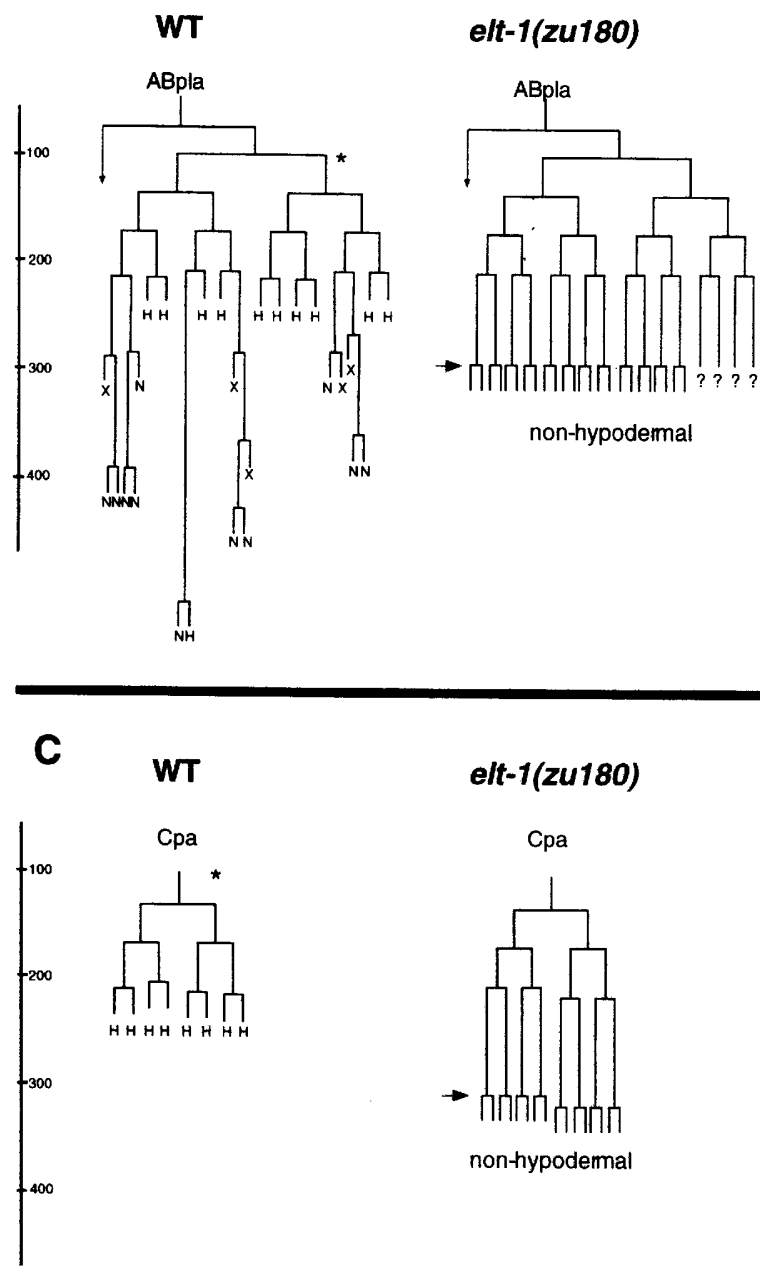

elt-1(zu180)

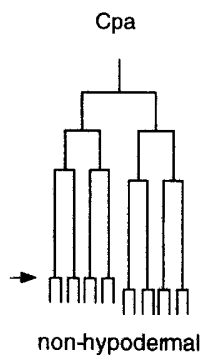

daughter becomes a hypodermal cell and the small daughter becomes either a neuroblast or a neuronal support cell (Sulston et al. 1983). In elt-1 mutants, these four ABarp descendants divide equally.

In a wild-type embryo, 22 of the ABarp descendants at
Figure 5. Lineage differences between wild-type and elt-1 mutant embryos. Examples are given of three representative lineages, ABarp, ABpla, and Cpa in wild-type and elt-1 embryos. The horizontal lines on the lineage diagrams indicate cell divisions and the vertical axes represent time between divisions. Time 0 is at fertilization. Cell divisions observed in elt $-1 \mathrm{em}$ bryos that are not present in wild-type embryos are indicated by arrows. Note that divisions that are unequal in wild type, as indicated by $<$ or $>$ signs, are equal $|=|$ in elt -1 . These data are a summary from six elt-1 embryos. Not all cells could be followed in each embryo; all lineage data presented here were observed in at least two embryos. (S) Neuronal support cell; $(\mathrm{H})$ hypodermal cell; (N) neuron; (X) cell death; (?) undetermined. Data for wildtype lineages taken from Sulston et al. (1983).

220 min begin to flatten and spread, differentiating as hypodermal cells; these cells do not divide again during embryogenesis (Fig. 5A). In elt-1 mutants, these ABarp descendants remain rounded instead of flattening, and undergo at least one additional division, producing cells with a neuronal morphology (Fig. 5A; additional divisions indicated by arrow). The extra cell divisions in elt-1 mutants occur at a time when several blastomeres in wild-type embryos are dividing to produce neurons (Sulston et al. 1983). The abnormal ABarp lineage in elt-1 mutants, however, is not identical in detail to the lineage pattern of any wild-type blastomere.

To determine whether the additional descendants of ABarp were becoming neurons in elt-1 mutants, we asked if these descendants express the neuronal marker H2O-GFP. This marker consists of a neuronal-specific promoter fused to a gene encoding the green fluorescent protein (GFP) with a nuclear localization signal; wildtype nematodes carrying the H2O-GFP transgene show fluorescence in most neurons (T. Ishihara and I. Katsura, pers. comm.; see Materials and Methods). We examined the expression of $\mathrm{H} 2 \mathrm{O}-\mathrm{GFP}$ in operated embryos in which all blastomeres except ABarp and ABalp were killed with a laser microbeam. In wild-type development, cell-cell interactions cause ABalp to adopt a different fate from that of ABarp (Hutter and Schnabel 1994; Mello et al. 1994). Our ablation strategy, however, prevents these interactions such that both ABalp and ABarp adopt the fate of ABarp. We found that the laser-operated wild-type embryos produce many hypodermal cells as expected, and only about seven H2O-GFP-positive neurons. In contrast, operated elt-1 mutants produce an average of $28 \mathrm{H} 2 \mathrm{O}-\mathrm{GFP}$-positive neurons, and no cells resembling hypodermal cells (Fig. 4C,D; Table 1). Thus, we conclude that elt-1 function is necessary for the wildtype development of ABa-derived hypodermal cells and that loss of elt-1 activity leads to this blastomere producing neurons instead of hypodermal cells.

$A B p$-derived hypodermal precursors are transformed in elt-1 mutants

The ABp-derived blastomeres ABpra and ABpla produce a total of 36 major hypodermal cells in wild-type em- 
Page et al.

Table 1. Number of neurons produced by isolated blastomeres

\begin{tabular}{lcr}
\hline Blastomeres & Wild type & elt-1(zu180) \\
\hline ABarp and ABalp & $7 \pm 2\{n=48\}$ & $28 \pm 7(n=17)$ \\
C & $0 \pm 0\{n=15)$ & $0 \pm 0(n=11)$ \\
\hline
\end{tabular}

Blastomeres were isolated, allowed to develop, and examined for $\mathrm{H} 2 \mathrm{O}-\mathrm{GFP}$ expression. Details of blastomeres isolation are in Materials and Methods.

bryos. We examined the development of these blastomeres in elt-1 mutants and found that they have abnormal patterns of cell division, similar to that of $A B a r p$, the $\mathrm{ABa}$-derived blastomere (Fig. 5, cf. B to A). ABp descendants that become hypodermal cells in wild-type embryos undergo an extra division in elt-1 mutants, and the cells produced by these extra divisions do not resemble hypodermal cells. We isolated the ABp blastomere by laser-ablation experiments (see Materials and Methods) and examined the amount of neuronal tissue produced by $\mathrm{H} 2 \mathrm{O}-\mathrm{GFP}$ expression. In contrast to $\mathrm{ABa}, \mathrm{ABp}$ does not appear to produce extra neurons in the elt-1 mutant. In similar experiments we tested whether $\mathrm{ABp}$ produces muscle cells instead of hypodermal cells in elt-1 mutants by use of the muscle-specific marker hlh-1-GFP (K. Dej, S. Xug, and A. Fire, pers. comm.), and found that ABp does not produce extra muscle cells. We conclude that elt-1 is required for the specification of major hypodermal cells from the ABp blastomere. In elt-1 mutants, however, $\mathrm{ABa}$ and $\mathrm{ABp}$ descendants have different transformations in cell fate.

\section{C-derived hypodermal precursors become muscle precursors in elt-1 mutants}

In wild-type development, the $\mathrm{C}$ blastomere produces 13 major hypodermal cells and 32 muscle cells (Sulston et al. 1983). Two C granddaughters, Cpa and Caa, almost exclusively produce hypodermal cells, whereas the other two granddaughters, Cpp and Cap, produce only muscle cells. We examined the lineage of C in elt-1 mutants and found that both $\mathrm{Cpa}$ and $\mathrm{Ca}$ descendants undergo an additional round of division, producing cells that do not resemble hypodermal cells in morphology (Fig. 5C shows the lineage of Cpa in both wild-type and elt-1 mutant embryos). To test whether the abnormal $\mathrm{Cpa}$ and $\mathrm{Caa}$ descendants are becoming neurons, we examined the expression pattern of the neuronal marker H2O-GFP in laser-operated embryos in which only the $\mathrm{C}$ blastomere was allowed to develop. In an operated wild-type embryo, the $\mathrm{C}$ blastomere produces numerous hypodermal cells, but no H2O-GFP-positive cells (Table 1). We found that the $\mathrm{C}$ blastomere in an operated elt-1 embryo does not appear to produce hypodermal cells but also does not produce $\mathrm{H} 2 \mathrm{O}-\mathrm{GFP}$-positive cells (Table 1). These results suggest that $C$ does not produce extra neurons in elt 1 mutants.

To test the possibility that the abnormal Cpa and Caa descendants become muscle cells instead of hypodermal cells, we examined the expression of the muscle marker
hlh-1-GFP in elt-1 mutants. To examine the development of only Cpa and Caa, we killed all other blastomeres with a laser microbeam. Cpa and Caa blastomeres from wild-type embryos $(n=19)$ produce hypodermal cells but do not produce muscle cells, as expected. In contrast, when either Cpa or Caa is isolated in elt-1 mutants ( $n=3$ and 5, respectively), each blastomere produces an average of 13 muscle cells. Thus, $\mathrm{C}, \mathrm{ABa}$, and $\mathrm{ABp}$ blastomeres all require elt-1 to produce hypodermal cells. In the absence of elt-1 function, however, C produces muscle cells instead of hypodermal cells while $\mathrm{ABa}$ produces neurons.

\section{Cell fate transformations in elt-1 mutants are affected by lineage-specific factors}

Why do C-derived hypodermal precursors become muscle precursors in elt-1 mutants while the $\mathrm{ABa}$ and $\mathrm{ABp}$-derived precursors adopt nonmuscle fates? The Cderived precursors in elt-1 mutants could respond to muscle-determining factors that are normally suppressed by elt-1|+| activity. These could be position-specific extrinsic factors (Schnabel 1995), or lineage-specific intrinsic factors (Hunter and Kenyon 1996). To ask whether the posterior positions of C-derived precursors affect their pattern of development in elt-1 mutants, we constructed and examined mex-3;elt-1 double mutants. In mex-3 mutants, the lineage-specific transcription factor PAL-1 is misexpressed in AB descendants, causing these anterior blastomeres to adopt C-like fates and produce hypodermal cells and muscle cells (Fig. 6C; Draper et al. 1996; Hunter and Kenyon 1996). If the transformed fates of hypodermal cells in the elt-1 mutant are caused by position-specific factors, many of these anterior blastomeres in mex-3;elt-1 mutants would produce neurons, as do $\mathrm{ABa}$ descendants in elt-1 mutants. If the transformed fate depends on lineage-specific factors, however, these anterior blastomeres would be expected to produce muscle cells, as do C descendants in elt-1 mutants. We found that the anterior region of the mex-3;elt-1 double mutant lacks hypodermal cells, and instead consists almost entirely of muscle cells (Fig. 6E) with very few neurons (Fig. 6F).

\section{ELT-1 is expressed in hypodermal cells and their precursors}

To determine when and where the ELT-1 protein is expressed, antibodies were generated against two regions outside the zinc finger domains of ELT-1 (see Materials and Methods). Antisera against both regions of ELT-1 give identical staining patterns. This pattern is not detected in wild-type embryos stained with preimmune sera, or in elt-1(zu180) mutants stained with anti-ELT-1 sera (see Materials and Methods). The 71 major hypodermal cells are born $\sim 260$ min postfertilization (about the 365-cell stage); these cells are readily identified in embryos stained for adherens junctions (Fig. 7B). At this stage, ELT-1 is detected in the nuclei of all 71 major hypodermal cells, and is not detected in any other cell type (Fig. 7A,B). Two categories of cells that do not stain 


\section{body-wall muscle}
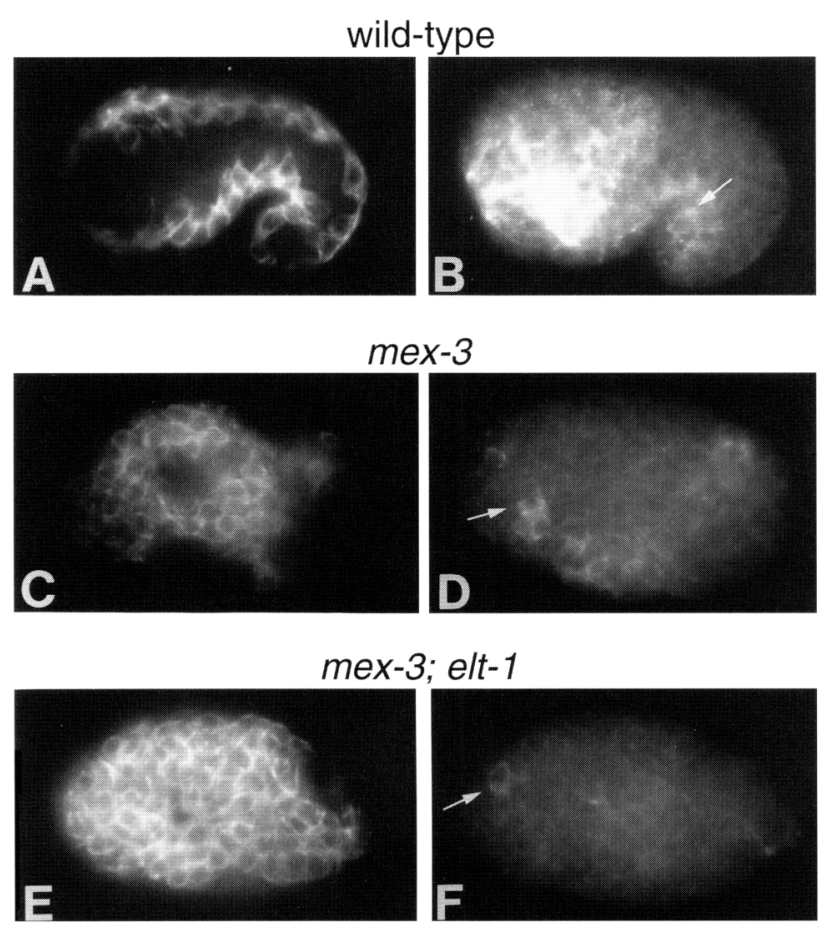

Figure 6. Immunofluorescence micrographs of body-wall muscle cells (left) and neurons (right) in wild-type $(A, B \mid$, mex-3 $\mid C, D)$, and mex-3;elt-1 $(E, F)$ embryos. Embryos $6 \mathrm{hr}$ after fertilization at $22^{\circ} \mathrm{C}$ were fixed and stained with $\mathrm{mAb} 5.6$ to visualize body-wall muscle cells and with anti-UNC-33 to visualize neurons. mex- 3 embryos generate more muscle cells $(C)$, but produce less neurons $(D)$, than do wild-type embryos $(A$ and $B$, respectively). mex-3;elt-1 embryos produce even more muscle cells than mex-3 single mutants (cf. $E$ with $C$ ) and only a few neurons $(F \mid$. Arrows point to individual neurons; a neuron has cytoplasmic staining surrounding the nonstaining nucleus.

for ELT-1 should be noted. First, ELT-1 is not detected in the nuclei of minor hypodermal precursors, and is not detected in the nuclei of their descendants later in embryogenesis. Second, ELT-1 is not detected in the nuclei of the nonhypodermal sisters of major hypodermal cells. As the embryo undergoes morphogenesis, ELT-1 levels appear to decrease in the dorsal and ventral hypodermal nuclei but persist at relatively high levels in the lateral, or seam, nuclei (Fig. 7C,E,G); ELT-1 can be detected in these hypodermal seam nuclei throughout the remainder of embryogenesis (data not shown).

The ELT-1 protein first is detected at $\sim 100 \mathrm{~min}$ postfertilization at the 28-cell stage of embryogenesis. ELT-1 is detected in the nuclei of all four $\mathrm{C}$ granddaughters but is not detected in any other nuclei (data not shown). Thus, at the 28-cell stage, ELT-1 is present in the two C granddaughters that produce hypodermal cells as well as the two granddaughters that produce only muscle cells. In subsequent stages, ELT-1 can no longer be detected in the C-derived muscle precursors, but is detected in the C-derived hypodermal precursors. In the 44-cell embryo, ELT- 1 is detected in the C-derived hypodermal precur- sors and in a subset of the $32 \mathrm{AB}$ descendants (Fig. 8A). The developmental time at which staining first is detected for each hypodermal lineage is indicated by an asterisk beside the wild-type lineages in Figure 5 . Because there is natural variability in the position of cells at the 44-cell stage (Schnabel et al. 1997) and because additional variability is introduced by our fixation technique, we have not yet been able to identify all of the $A B$ descendants at this stage with certainty. However, the positions and number of cells that express ELT-1 at this stage correlate well with the positions and number of hypodermal precursors present at this stage (Table 2; Sulston et al. 1983). ELT-1 is expressed in the AB descendants through the successive rounds of cell division until
ELT-1
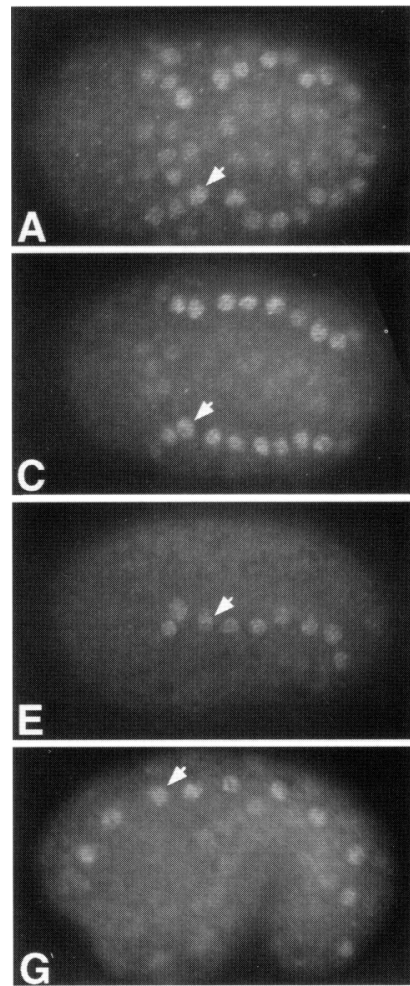

adherens junctions
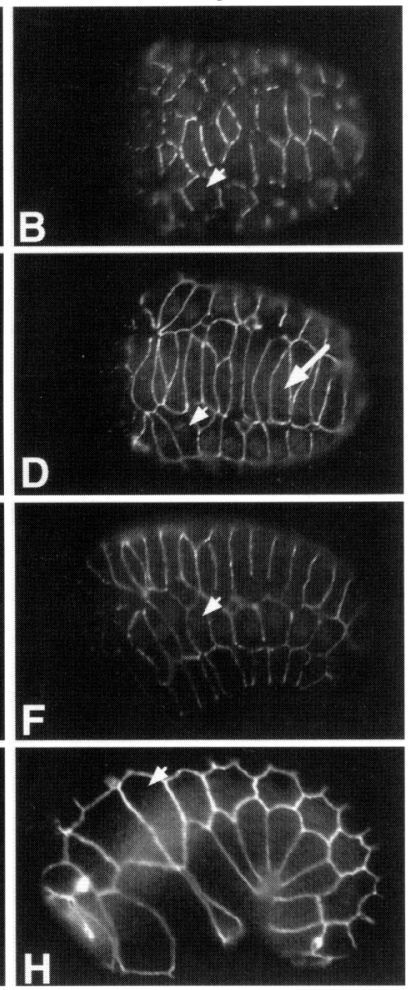

Figure 7. Expression pattern of the ELT-1 protein midway through embryonic development. Immunofluorescence micrographs of wild-type embryos stained with anti-ELT-1 sera (left), and with mAb MH27 (right) to outline major hypodermal cells. $(A, B)$ Dorsal view of an embryo $\sim 260$ min postfertilization. The major hypodermal cells have just been born, and the majority of these cells reside on the dorsal surface. ELT-1 is present in the nuclei of all major hypodermal cells at this stage. Note the absence of ELT-1 staining in the anterior neuronal precursors (cf. 1A). The short arrows point to the nucleus of a seam cell in $A-H$. $(C, D)$ Dorsal view of an embryo 300 min postfertilization. A hypodermal cell in the dorsal row is indicated by the long arrow in $D$. ELT-1 staining is strongest in the seam cell nuclei. $(E, F)$ Lateral view of an embryo at a similar stage to that shown in $C$ and $D$. The ventral row of hypodermal cells have very faint nuclear staining. $(G, H)$ Lateral view of an embryo $\sim 400$ min postfertilization. 


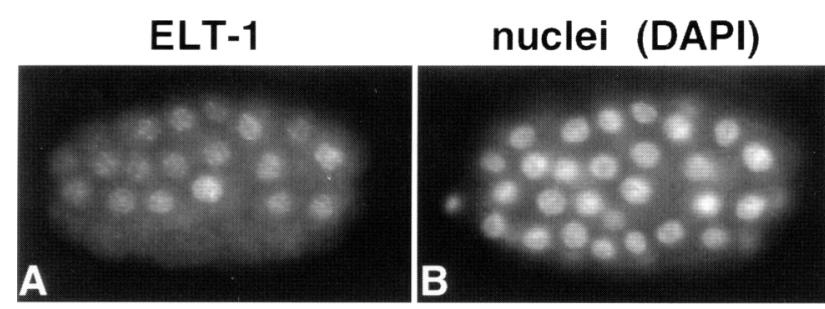

Figure 8. Expression pattern of the ELT-1 protein at the 51-cell stage. Dorsal/lateral view of a wild-type embryo stained with anti-ELT-1 $(A)$ and with DAPI $(B)$ to visualize nuclei. Dorsal/ lateral, but not ventral, nuclei show high levels of ELT-1.

the terminal division of the hypodermal cells (Fig. 7A). Thus, ELT-1 is expressed in blastomeres that generate major hypodermal cells and in the major hypodermal cells.

\section{Discussion}

elt-1 functions in the specification of the hypodermal cell fate

In this study we have shown that the elt-1 gene, which encodes a GATA-like transcription factor, is essential for the development of major hypodermal cells in the $C$. elegans embryo. elt 1 mutants lack cells with morphological and molecular characteristics of major hypodermal cells: In elt-1 mutant embryos, no cells resembling major hypodermal cells are visible by light microscopy, or are detected with antibodies that recognize the adherens junctions of hypodermal cells. elt-1 mutants also fail to express a marker specific for seam cells, the lateral group of major hypodermal cells. Other cell types, such as pharyngeal, intestinal, neuronal, and muscle cells are present in elt-1 mutants, indicating that elt-1 mutations do not affect the development of most other tissues.

elt-1 may not be required for the development of the minor hypodermal cells that normally form the specialized hypodermis at the termini of the body. elt-1 mutants contain a few small cells of undetermined identity that are surrounded by adherens junctions. These cells may be minor hypodermal cells, but alternatively could be neuronal support cells that contain adherens junctions and that normally link to the hypodermis. ELT-1 protein is not detected in minor hypodermal cells or their precursors in wild-type embryos, consistent with the hypothesis that elt-1 activity is not required for the development of these cells.

In principle, genes required for hypodermal development could function at one of several distinct steps in embryogenesis. Such genes could function at the beginning of embryogenesis to determine the identity of early blastomeres that ultimately produce hypodermal cells, they could function later in development to specify the hypodermal cell fate, or they could be required simply to maintain the differentiated state of hypodermal cells. Several maternally expressed genes have been described in C. elegans that function in specifying the identity of early blastomeres (Priess 1994). Mutations in these genes often cause the affected blastomere to adopt a pattern of cleavage and differentiation similar, or identical, to that of a blastomere located elsewhere in the embryo. For example, mutations in the gene $g l p-1$ can cause ABp to adopt a pattern of development characteristic of $\mathrm{ABa}$ (Hutter and Schnabel 1994; Mango et al. 1994; Mello et al. 1994, Moskowitz et al. 1994). In elt-1 mutant embryos, the ABarp, ABpla, and ABpra blastomeres have abnormal lineages; however, these lineage patterns are not identical to any wild-type lineage pattern. Although these blastomeres fail to generate hypodermal cells, other cell types normally generated by these blastomeres are present in elt-1 embryos. In normal embryogenesis, ABarp, ABpla, and ABpra blastomeres produce major hypodermal cells and are the only blastomeres that produce the four specialized touch neurons. In elt-1 mutant embryos, these four touch neurons are present (B. Page, unpubl.), indicating that elt-1 is required for only a subset of the cell types generated by these blastomeres.

We propose that elt-1 activity is required for the specification, rather than for the differentiation or maintenance, of major hypodermal cells. In contrast, the gene lin-26, which also is required for hypodermal development in C. elegans embryos, appears to function after the hypodermal cell fate is specified. Cells with several characteristic features of hypodermal cells are produced in lin-26 mutant embryos, however, these cells appear to degenerate (Labouesse et al. 1994). We have shown that mutations in elt-1 cause precursors that normally produce hypodermal cells to produce neurons or muscle cells instead. This result suggests that in wild-type embryogenesis these precursors are not irrevocably committed to the hypodermal fate prior to elt-1 function. Thus, elt-1 likely functions at a time when blastomeres are pluripotent, as expected for a gene involved in specifying cell fate. Molecular studies support the hypothesis that elt-1 functions upstream of lin-26. Chromosomal deficiencies of the elt-1 region have been shown to prevent the wild-type expression pattern of the LIN-26 protein (Chanal and Labouesse 1997), as do mutations in elt-1 itself (B.P. unpubl.).

When does elt-1 function to specify the hypodermal cell fate? The ELT-1 protein is detected in differentiated hypodermal cells as well as in precursors of hypodermal cells. We have evidence that in some hypodermal lineages elt $-1|+|$ functions before the birth of the hypodermal cell. In wild-type embryos, these lineages generate hypodermal cells by an unequal cell division; the large daughter differentiates as a hypodermal cell and the

Table 2. Number of ELT-1-positive cells compared with the number of maior hypodermal precursors

\begin{tabular}{lccc}
\hline $\begin{array}{l}\text { Approximate } \\
\text { developmental } \\
\text { time (min) }\end{array}$ & $\begin{array}{c}\text { Total } \\
\text { number } \\
\text { of cells }\end{array}$ & $\begin{array}{c}\text { ELT-1-positive } \\
\text { cells }\end{array}$ & $\begin{array}{c}\text { Hypodermal } \\
\text { precursors }\end{array}$ \\
\hline 100 & $51 \pm 5$ & $18 \pm 4(n=6)$ & 14 \\
170 & $85 \pm 8$ & $22 \pm 5(n=9)$ & 23 \\
\hline
\end{tabular}

Developmental time after fertilization at $20^{\circ} \mathrm{C}$. The number of hypodermal precursors is predicted from the wild-type lineage (Sulston et al. 1983). 
small daughter adopts a nonhypodermal cell fate. In elt-1 mutants, these divisions produce daughters of equal size, indicating that elt-1 functions at or prior to these divisions. Thus, elt-1 may function within a few cell divisions before the hypodermal cells are born.

\section{Patterns of cell differentiation in elt-1 mutants}

We propose that $\mathrm{ABa}, \mathrm{ABp}$, and $\mathrm{C}$ descendants adopt different nonhypodermal fates in elt-1 mutants because these blastomeres normally generate hypodermal cells by distinct genetic pathways. We have shown that in elt-1 mutants, the $\mathrm{C}$ blastomere generates muscle cells instead of hypodermal cells, whereas the ABa blastomere generates neurons instead of hypodermal cells. These results indicate that hypodermal specification in the $\mathrm{C}$ lineage normally may involve a choice between hypodermal and muscle cell fates, whereas hypodermal specification in the ABa lineage may involve a choice between hypodermal and neuronal cell fates.

The maternally expressed gene pal-1 appears to promote both hypodermal and muscle fates in $\mathrm{C}$ descendants (Hunter and Kenyon 1996). The PAL-1 protein, a transcription factor related to Drosophila CAUDAL, is expressed in $\mathrm{C}$ descendants, but not $\mathrm{AB}$ descendants. If PAL-1 is expressed inappropriately in $A B$ descendants, as is the case in mex-3 mutants, $A B$ descendants adopt a cell fate similar to $\mathrm{C}$ and produce muscle cells and hypodermal cells (Draper et al. 1996). We have shown here that $\mathrm{AB}$ produces muscle cells instead of hypodermal cells in mex-3;elt-1 double mutants, as does the C blastomere in elt-1 mutants.

At present, we do not know what cell type the ABp blastomere generates instead of hypodermal cells in elt-1 mutants. These nonhypodermal cells, however, do not appear to be muscle cells or neurons. Why is the cell fate transformation of $\mathrm{ABp}$ descendants different from that of $\mathrm{ABa}$ in elt -1 mutants? ABp undergoes a GLP-1/APX-1 mediated cell interaction that $\mathrm{ABa}$ does not; this interaction results in $\mathrm{ABp}$ undergoing a pattern of development that differs from $\mathrm{ABa}$ (Mango et al. 1994; Mello et al. 1994). In glp-1;elt-1 mutants, we find that ABp produces excess neurons instead of hypodermal cells, a cell fate transformation similar to $\mathrm{ABa}$ in elt-1 single mutants (B. Page, unpubl.). Mutations in $g l p-1$ and mex-3 have no affect on the positions of early embryonic blastomeres. Thus, the different transformations in cell fate seen in elt-1 mutants are a consequence of the different pathways used to establish the identities of the early blastomeres.

\section{Control of elt- 1 expression}

ELT-1 protein is detected in the nuclei of a contiguous group of $\mathrm{ABa}, \mathrm{ABp}$, and $\mathrm{C}$ descendants on the dorsal surface of early embryos. The similar positions of the cells that express ELT-1 suggest the possibility that simple positional cues could contribute to elt-1 expression. Our current understanding of cell type specification in C. elegans, however, suggests that lineage-specific mechanisms could also determine gene expression patterns. For example, the homeobox gene mab-5 normally is expressed in a simple pattern in the posterior of the embryo (Costa et al. 1988). Yet, even if cells that normally express mab-5 are mispositioned to the anterior of the embryo, these cells still express mab-5 (Cowing and Kenyon 1996). Little is known at present about how complex, lineage-specific patterns of expression are achieved in the C. elegans embryo. Promoter analysis of the musclespecific hlh-1 gene, which is expressed in multiple lineages, suggests that distinct cis-acting promoter elements can activate $h l h-1$ expression in a lineage-dependent manner (Krause et al. 1994). It will be of interest to determine whether the expression of the elt-1 gene is regulated similarly by separate, lineage-specific elements. For example, elt-1 expression in the $C$, but not $\mathrm{ABa}$ or $\mathrm{ABp}$, lineage might be directly regulated by $\mathrm{PAL}-$ 1 , since expression of PAL-1 appears to precede and overlap with that of ELT-1 in C descendants.

Part of the simple pattern of ELT-1 expression on the dorsal surface of the embryo requires the loss of ELT-1 expression in cells that leave the dorsal surface during development. We have observed that all four granddaughters of the C blastomere initially express ELT-1. ELT-1 expression is maintained in the descendants of the two C granddaughters that are hypodermal precursors; these descendants remain on the dorsal surface of the embryo. In contrast, the two $\mathrm{C}$ granddaughters that are muscle precursors produce descendants that leave the surface and that lose ELT-1 expression. It should be possible to test in future experiments whether loss of elt-1 expression is a prerequisite for these $\mathrm{C}$ descendants to become internalized and to differentiate as muscle.

Although the early embryonic development of C. elegans and Drosophila seems very different at the cellular level, there is evidence of some common molecular features. The posterior-specific PAL-1 protein is closely related to the posterior-specific CAUDAL protein in Drosophila (Waring and Kenyon 1991; Hunter and Kenyon 1996). Similarly, the VAB-7 protein, which is required for proper embryonic patterning in some lineages in C. elegans is related to the pair-rule EVE protein in Drosophila (Ahringer 1996) In this respect, it is intriguing that the elt-1 gene of $C$. elegans and the pannier gene of Drosophila encode similar GATA-like transcription factors (Ramain et al. 1993). Wild-type Drosophila embryos express high levels of the pannier mRNA in the dorsal ectodermal cells (Winick et al. 1993). Severe mutations in pannier cause embryonic lethality, and the arrested embryos appear to lack epidermal cells. It is thus possible that pannier and elt-1 have evolutionarily related functions in epidermal tissue specification. It will be interesting to determine whether other components of the pathways leading to hypodermal/epidermal specification are similar in the two organisms.

\section{Materials and methods}

\section{Strains and alleles}

The Bristol strain N2 was used as the standard wild-type strain. The genetic markers and deficiencies used in this paper are 
listed by chromosome as follows: linkage group I (LGI): bli3(e767), ced-1(e1735), egl-30(ad805), mex-3(zu166). LGIV: daf14(m77), dpy-13(e184), dpy-20(e1282), emb-11(g4), fem3(e1996), let-51(s41), let-55(s45), let-657(s1254), unc-24(e138), unc-43(e408), eDf18, eDf19, mDf7, nT1. LGX: lin-2(e1309). mex-3(zu166) was provided by B. Draper (this laboratory). The strain JR126 contains a seam-cell specific lac Z fusion expression construct that is integrated into the genome /Gendreau et al. 1994); this strain was provided by J. Rothman (University of California, Santa Barbara). The H2O-GFP neuronal marker used in this study was designed and integrated into the genome by $\mathrm{T}$. Ishihara and I. Katsura (National Institute of Genetics, Mishima, Japan). All other mutant strains listed were obtained or are available from the C. elegans Genetic Stock Center. The basic methods for worm culture and genetics were performed as described by Brenner (1974).

\section{Isolation of elt- 1 alleles}

The elt-1(zu180), (zu220), and (zu228) mutations were isolated independently in screens for nonconditional embryonic-lethal mutations. lin-2 mutant larvae were used as the starting strain for mutagenesis as described previously (Priess et al. 1987), except that $F_{1}$ animals were scored for dead eggs instead of $F_{2}$ animals. Larvae were placed on microscope slides and viewed by Nomarski optics to score tissue differentiation in the inviable embryos.

\section{Genetic analysis}

elt-1(zu180) was positioned on chromosome IV by standard linkage tests (Brenner 1974). zu220 and zu228 were shown to be alleles of elt-1 by complementation tests. elt-1(zu180) was mapped to the right arm of chromosome IV between fem- 3 and daf-14 by standard three- or four-factor analysis. The mapping data are available from the $C$. elegans stock center.

\section{Cloning of elt-1}

The genomic DNA between fem-3 and daf-14 has been cloned and ordered into overlapping sets of cosmids and yeast artificial chromosomes (Coulson et al. 1986; 1988). Tom Barnes generously sent us a collection of strains carrying extrachromosomal arrays that were generated by coinjection of cosmids in the fem-3 daf-14 region. These arrays were crossed into the strain elt-1(zu180)unc-43(e408)/unc-24(e138)dpy-20(e1282). One set of arrays rescued the embryonic lethal phenotype of elt1(zu180). This array was generated by the injection of the following collection of cosmids: F13C8, C48A2, R05H9, C33A12, T01G2, T12H5, F38E11, F56H11, and T05Al. We microinjected these cosmids individually into the gonads of elt-1(zu180)unc43(e408)/unc-24(e138)dpy-20(e1282) hermaphrodites using the procedures of Mello et al. (1991).

\section{ELT-1 immunostaining}

Rabbit polyclonal antibodies were raised against two different ELT-1-specific peptides: (ELT-1C) ELDQSGVWGMKNTQPMLMTPT and (ELT-1D) SNFYFNSIEDQLEYKTC. Embryos were fixed and stained as described previously (Lin et al. 1995). Antisera against both peptides give identical staining patterns. To examine the staining pattern in elt-1(zu180) mutants, embryos of various stages were stained with anti-ELT-1. The hypodermal cells stained by anti-ELT-1 in wild-type embryos are absent in terminal stage elt-1 embryos. Thus, we examined early embryos (100-200 cells) for ELT-1 staining prior to hypo- dermal cell differentiation. Only $4 \%$ of embryos isolated from $\mathrm{N} 2$ hermaphrodites lacked anti-ELT-1 staining ( $n=71)$. In contrast, $23 \%$ of embryos from elt-1(zu180) heterozygous hermaphrodites, lacked anti-ELT-1 staining ( $n=112)$. The $23 \%$ of embryos not stained with anti-ELT-1 correlates with the $25 \%$ expected to be elt-1(zu180) homozygotes.

\section{Analysis of embryos}

A Zeiss Axioplan microscope equipped with DIC (Nomarski) optics and epifluorescence was used for microscopy. Embryos were prepared for light microscopy following the method of Sulston et al. (1983). Embryos were processed for fluorescence microscopy as described in Bowerman et al. (1992, 1993). Photographs were taken with Kodak Technical Pan film and developed in Kodak HCl10 developer. Laser ablation experiments were performed at $22^{\circ} \mathrm{C}$ with a VSL-337 laser attached to a Zeiss Axioscope microscope as described in Avery and Horvitz (1989). The procedure for isolating ABarp and $\mathrm{ABalp}$ was as follows: at the 4-cell stage, blastomeres $\mathrm{ABp}, \mathrm{EMS}$, and $\mathrm{P}_{2}$ were killed with a laser microbeam. Subsequently, $\mathrm{ABa}$ underwent a left/right division and these descendants, $\mathrm{ABal}$ and $\mathrm{ABar}$, divided anterior/posterior. The two anterior granddaughters of $\mathrm{ABa}$ then were killed with a laser microbeam. The blastomere $\mathrm{C}$ was isolated by killing $\mathrm{ABa}, \mathrm{ABp}$, and EMS at the 4-cell stage. After $\mathrm{P}_{2}$ divided into $C$ and $P_{3}, P_{3}$ was killed. In all of these experiments, the nonablated blastomeres were allowed to develop for $20 \mathrm{hr}$ at $15^{\circ} \mathrm{C}$, and the number of cells expressing the H2O-GFP marker was determined by use of epifluorescence. Lineage analysis was performed with the four-dimensional system described in Draper et al. (1996).

\section{Acknowledgments}

We thank M. Costa, R. Hill, and K. Mickey for critical reading of this manuscript and helpful suggestions, and R. Hill for providing assistance with the lineage analysis. We thank K. Dej, S. $\mathrm{Xug}$, and A. Fire for the muscle-specific marker hlh-1-GFP; I. Katsura and $\mathrm{T}$. Ishihara for the neuronal-specific marker $\mathrm{H} 2 \mathrm{O}-$ GFP; J. Shaw for the UNC-33 antibody; and J. Rothman for the strain JR126. We thank the Caenorhabditis Genetics Center for providing several nematode strains. We are especially grateful to Tom Barnes for providing transgenic strains. B.D.P. was supported by a postdoctoral training grant (GM16183-02) from the National Institutes of Health (NIH), and K.S. was supported by the Medical Research Council (Canada). This research was supported by grants from NIH to T.B. (GM30870) and J.R.P. and by the Howard Hughes Medical Institute (J.R.P.).

The publication costs of this article were defrayed in part by payment of page charges. This article must therefore be hereby marked "advertisement" in accordance with 18 USC section 1734 solely to indicate this fact.

\section{References}

Ahringer, J. 1996. Posterior patterning by the Caenorhabditis elegans even-skipped homolog vab-7. Genes \& Dev. 10: 1120-1130.

Albertson, D.G. and J.N. Thomson. 1976. The pharynx of Caenorhabditis elegans. Philos. Trans. R. Soc. Lond. B Biol. Sci. 275: 299-325.

Avery, L. and H.R. Horvitz. 1989. Pharyngeal pumping continues after laser killing of the pharyngeal nervous system of $C$. elegans. Neuron 3: 473-485.

Bowerman, B., B.A. Eaton, and J.R. Priess. 1992. skn-1, a mater- 
nally expressed gene required to specify the fate of ventral blastomeres in the early C. elegans embryo. Cell 68: 10611075.

Bowerman, B., B.W. Daper, C.C. Mello, and J.R. Priess. 1993. The maternal gene $s k n-1$ encodes a protein that is distributed unequally in early $C$. elegans embryos. Cell 74: 443452.

Brenner, S. 1974. The genetics of Caenorhabditis elegans. Genetics 77: 71-94.

Chanal, P. and M. Labouesse. 1997. A screen for genetic loci required for hypodermal cell and glial-like cell development during Caenorhabditis elegans embryogenesis. Genetics (in press!.

Costa, M., M. Weir, A. Coulson, J. Sulston, and C. Kenyon. 1988. Posterior pattern formation in the C. elegans involves position-specific expression of a gene containing a homeobox. Cell 55: 747-756.

Coulson, A.J., J. Sulston, S. Brenner, and J. Karn. 1986. Towards a physical map of the genome of the nematode Caenorhabditis elegans. Proc. Natl. Acad. Sci. 83: 7821-7825.

Coulson, A., R. Waterston, J. Kiff, J. Sulston, and Y. Kohara. 1988. Genome linking with yeast artificial chromosomes. Nature 335: 184-186.

Cowing, D. and C. Kenyon. 1996. Correct Hox gene expression established independently of position in Caenorhabditis e1egans. Nature 382: 353-356.

Draper, B.W., C.C. Mello, B. Bowerman, J. Hardin, and J.R. Priess. 1996. MEX-3 is a KH domain protein that regulates blastomere identity in the early $C$. elegans embryos. Cell 87: 205-216.

Evans, T.C., S.L. Crittenden, V. Kodoyianni, and J. Kimble. 1994. Translational control of maternal $g l p-1$ mRNA establishes an asymmetry in the C. elegans embryo. Cell 77: 183194.

Gendreau, S.B., I.P.G. Moskowitz, R.M. Terns, and J. Rothman. 1994. The potential to differentiate epidermis is unequally distributed in the $\mathrm{AB}$ lineage during early embryonic development in C. elegans. Dev. Biol. 166: 770-781.

Guo, S. and K.J. Kemphues. 1995. par-1, a gene required for establishing polarity in C. elegans embryos, encodes a putative Ser/Thr kinase that is asymmetrically distributed. Cell 81: 611-620.

Hunter, C.P. and C. Kenyon. 1996. Spatial and temporal controls target pal-1 blastomere-specification activity to a single blastomere lineage in C. elegans embryos. Cell 87: 217-226.

Hutter, H. and R. Schnabel. 1994. glp-1 and inductions establishing embryonic axes in $C$. elegans. Development 120: 2051-2064.

— 1 1995. Specification of anterior-posterior differences within the $\mathrm{AB}$ lineage in the C. elegans embryo: A polarising induction. Development 121: 1559-1568.

Kemphues, K. and S. Strome. 1997. Fertilization and establishment of polarity in the embryo. In C. elegans $I I$ (ed. D. Riddle, T. Blumenthal, B. Meyer, and J. Priess), pp. 335-360. Cold Spring Harbor Laboratory Press, Cold Spring Harbor, NY.

Krause, M., S. Harrison, S.-Q. Xu, L. Chen, and A. Fire. 1994. Elements Regulating Cell- and Stage-Specific Expression of the C. elegans MyoD Family Homolog hlh-1. Dev. Biol. 166: $133-148$.

Labouesse, M., S. Sookhareea, and H.R. Horvitz. 1994. The C. elegans gene lin-26 is required to specify the fates of hypodermal cells and encodes a presumptive zinc-finger transcription factor. Development 120: 2359-2368.

Lin, R., S. Thompson, and J.R. Priess. 1995. pop-1 encodes an HMG box protein required for the specification of a mesoderm precursor in the early C. elegans embryos. Cell
83: 599-609.

Mango, S.E., C.J. Thorpe, P.R. Martin, S.H. Chamberlain, and B. Bowerman. 1994. Two maternal genes, apx-1 and pie-1, are required to distinguish the fates of equivalent blastomeres in the early Caenorhabditis elegans embryo. Development 120: 2305-2315.

Mello, C.C., J.M. Kramer, D. Stinchcomb, and A. Victor. 1991. Efficient gene transfer in C. elegans: Extra chromosomal maintenance and integration of transforming sequences. EMBO I. 10: 3959-3970.

Mello, C.C., B.W. Draper, and J.R. Priess. 1994. The maternal genes apx-1 and $g 1 p-1$ and establishment of dorsal-ventral polarity in the early C. elegans embryo. Cell 77: 95-106.

Moskowitz, I., S.B. Gendreau, and J.H. Rothman. 1994. Combinatorial specification of blastomere identity by $g l p$-1-dependent cellular interactions in the nematode Caenorhabditis elegans. Development 120: 3325-3338.

Orkin, S.H. 1992. GATA-binding transcription factors in hematopoietic cells. Blood 80: 575-581.

Priess, J.R. 1994. Establishment of initial asymmetry in early Caenorhabditis elegans embryos. Curr. Opin. Genet. Dev. 4: 563-568.

Priess, J.R. and D. Hirsh. 1986. Caenorhabditis elegans morphogenesis: The role of the cytoskeleton in the elongation of the embryo. Dev. Biol. 117: 156-173.

Priess, J.R. and J.N. Thomson. 1987. Cellular interactions in early C. elegans embryos. Cell 48: 241-250.

Priess, J.R., H. Schnabel, and R. Schnabel. 1987. The glp-1 locus and cellular interactions in early C. elegans embryos. Cell 51: 601-611.

Ramain, P., P. Heitzler, M. Haenlin, and P. Simpson. 1993. pannier, a negative regulator of achaete and scute in Drosophila, encodes a zinc finger protein with homology to the vertebrate transcription factor GATA-1. Development 119: 1277-1291.

Schnabel, R. 1995. Duels without obvious sense: Counteracting inductions involved in body wall muscle development in the C. elegans embryo. Development 121: 2219-2232.

Schnabel, R. and J. Priess. 1997. Specification of cell fates in the early embryo. In C. elegans $I I$ (ed. D. Riddle, T. Blumenthal, B. Meyer, and J. Priess), pp. 361-381. Cold Spring Harbor Laboratory Press, Cold Spring Harbor, NY.

Schnabel, R., H. Hutter, D. Moerman, and H. Schnabel. 1997. Assessing normal embryogenesis in the C. elegans embryo using a 4D-microscope: Variability of development and regional specification. Dev. Biol. 184: 234-256.

Spieth, J., Y.H. Shim, K. Lea, R. Conrad, and T. Blumenthal. 1991. elt-1, an embryonically expressed Caenorhabditis elegans gene homologous to the GATA transcription factor family. Mol. Cell. Biol. 11: 4651-4659.

Sulston, J., E. Schierenberg, J. White, and N. Thomson. 1983. The embryonic cell lineage of the nematode Caenorhabditis elegans. Dev. Biol. 100: 67-119.

Waring, D. A., and C. Kenyon. 1991. Regulation of cellular responsiveness to inductive signals in the developing C. elegans nervous system. Nature 350: 712-715.

Waterson, R.H. 1988. Muscle. In The nematode Caenorhabditis elegans (ed. W.B. Wood), pp. 281-335. Cold Spring Harbor Laboratory, Cold Spring Harbor, NY.

Winick, J., T. Abel, A. Michelson, I. Chardon-Loriaux, R. Homgren, T. Maniatis, and J.D. Engel. 1993. A GATA family transcription factor is expressed along the embryonic dorsoventral axis in Drosophila melanogaster. Development 119: 1055-1065.

Yochem, J. and I. Greenwald. 1989. glp-1 and lin-12, genes implicated in distinct cell-cell interactions in C. elegans, encode similar transmembrane proteins. Cell 58: 553-563. 


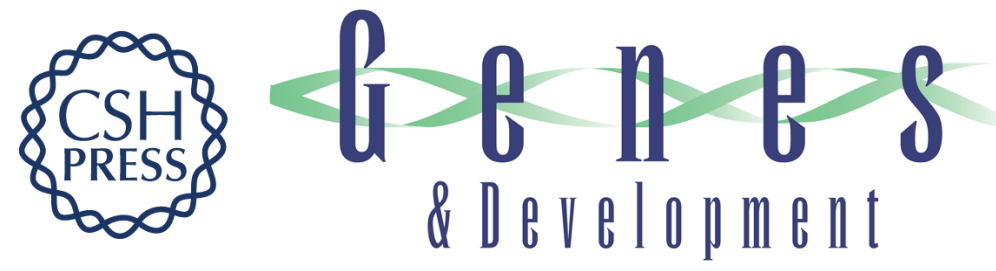

\section{ELT-1, a GATA-like transcription factor, is required for epidermal cell fates in Caenorhabditis elegans embryos.}

B D Page, W Zhang, K Steward, et al.

Genes Dev. 1997, 11:

Access the most recent version at doi:10.1101/gad.11.13.1651

References This article cites 37 articles, 13 of which can be accessed free at:

http://genesdev.cshlp.org/content/11/13/1651.full.html\#ref-list-1

License

Email Alerting

Service

Receive free email alerts when new articles cite this article - sign up in the box at the top right corner of the article or click here.

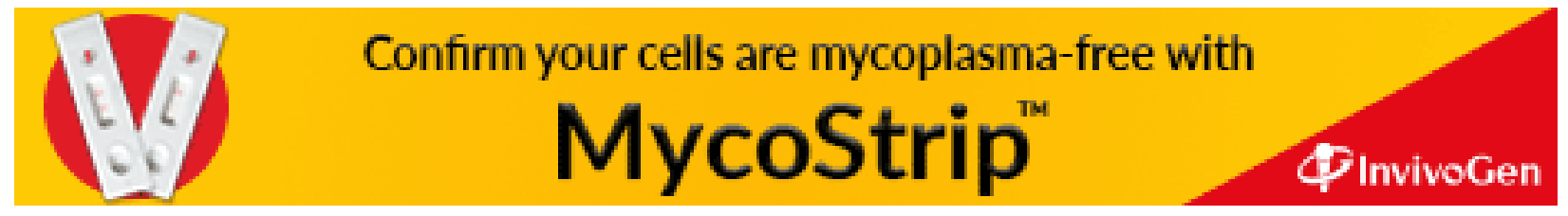

